\title{
Dynamics of Love and Happiness: A Mathematical Analysis
}

\author{
Dharna Satsangi \\ Department of Mathematics, Dayalbagh Educational Institute, Agra, India \\ Email: dharna.satsangi@gmail.com \\ Arun K. Sinha \\ Department of Mathematics, Dayalbagh Educational Institute, Agra, India \\ Email: arunsinha47@gmail.com
}

\begin{abstract}
The human behavior is a combination of several feelings. There are two cases whether a person likes other individual or not. The liking of an individual leads to love and finally happiness. The feelings of love may be in different forms but here considered to be partners' love. There are three aspects of love for the partner: forgetting process (oblivion), the pleasure of being loved (return), and the reaction to the appeal of the partner (instinct). Along with that the appeals and the personalities of the two individuals do not vary in time. This model proves that if the geometric mean reactive-ness to love is smaller than the geometric mean forgetting coefficient and the system is asymptotically stable if the ratio of appeals is greater than the reciprocal of ratio of mutual intensiveness coefficient.
\end{abstract}

Index Terms - Differential equations, Human behavior, Rowth-Hurwitz Criteria

\section{INTRODUCTION}

Today human behavior has become interesting topic to study. Everyone is looking for techniques to study it accurately. It is true that studying it is not difficult task. This can be performed by psychologists very easily but studying it accurately or near to accuracy is not easy one. It is clear that accuracy can be achieved by mathematical analysis of psychologists study. For this we need to define human behavior in form of equations or we need to form a model based upon different feelings taking account of different conditions. For example, love and hate are not two mutually exclusive events. A person can have both feelings simultaneously. Suppose a man likes some habits of his partner that are loved by him and some that are not liked are hated by him. So love and hate cannot be taken in different equations. Their combined effect on ones feelings shows his behavior.

There are different feelings in one's life; some of them are happiness and sadness. There are several factors affecting these feelings. Basically in mathematical terms sadness is negative feeling of happiness. If we assume ' $h$ ' is happiness factor then '- $h$ ' is sadness factor and everyone tries to achieve greater amount of ' $h$ '. Happiness can be achieved by external feelings. Among inner feelings love is the most dominant feeling in an individual. The feeling in an individual can be for his life, position, humanity or partner [1]. These feelings vary from person to person and from time to time. Everyone has these feelings in their hearts but the ratio or percentage differs. It is clear that the feeling in human varies according to different conditions, which are not easy to study mathematically. For it different conditions and assumptions have to be applied therefore we need to move towards an interpretative world or to think for a model that can give dynamics of human feelings and experiences specially happiness and love. There are some examples of love one is stories of Romeo Juliet and other is the creation of Tajmahal. Not even in the modern society but also in ancient time there were stories of Lord Krishna and Radha, Lord Krishna and Meera. The difference between these two is that one assumes Him true lover but other as her "everything" or she worships him. Also no one can ignore the phrase "God is Love" [2]. It is accepted in several religions. The Vedic literature describes the Absolute Truth as sat-cit-ananda vigraha, which means the personal form of eternal knowledge and bliss. And in the Taittiriya Upanishad (Second Valli, Seventh Anuvaka), the Absolute Truth is described as concentrated ananda or bliss. And the absolute truth is related with happiness which a man achieves whether it is worldly love or with the Supreme Being. The happiness is the emotion which is achieved either after getting your love or after completing a desire. So there is a direct relationship between love (desire) and happiness. Hence it is clear that happiness is proportional to love. As we get a relationship between the two quantities the mathematics come into the picture. Several scientists believed in this mathematics [3]. They have tried to bind the behavioral science with mathematics.

The rest of the paper is organized as follows. Initially existing research present in literature is presented in Section II. Here related models are explained. In Section III the model of consideration of this research which is an improvement of the existing model is presented. In Section IV the solution of the model is explained. In order to find weather the solution presented obeys stability conditions or not is tested in Section V. Finally Section VI concludes the paper. 


\section{EXISTING ART}

Ever since Newton introduced differential calculus, dynamical phenomena in Physics, Chemistry, Economics and all other sciences have been extensively studied by means of differential equations. Surprisingly, one of the most important problems concerning our lives, namely the dynamics of love between two persons, has been studied via system dynamics methodology. In his one page paper Strogatz [4] describes how the classical topic of harmonic oscillations can be taught to capture the attention of students. He suggested consideration of "a topic that is already on the minds of many college students: the time evolution of a love affair between two people". The model proposed by Strogatz is definitely unrealistic because it does not take into account the appeal of the two individuals. Thus Strogatz's model, though it layed the foundation of study of human romantic relationship, does not explain why two persons who are initially completely indifferent to each other develop a love affair.

In 1998, Rinaldi [5] in his paper proposed a more realistic, although it is still a minimal model. Three aspects of love dynamics are taken into account: the forgetting process (oblivion), the pleasure of being loved (return), and the reaction to the appeal of the partner (instinct). These three factors are assumed to be independent and are modeled by linear functions. The resulting model is a linear dynamical system, which turns out to be positive if the appeals of the two individuals are positive. The theory of positive linear systems can therefore be applied to this model and gives quite interesting results. Some of them describe the dynamic process of falling in love, i.e. the transformation of the feelings, starting from complete indifference (when two persons first meet) and tending towards a plateau. Other results are concerned with the influence that appeal and individual behavior have on the quality of romantic relationships.

Rinaldi considered three phenomenon namely, oblivion, return, and instinct. The first gives rise to a loss of interest in the partner, and explains, for example, the typical decay of $x_{i}$, which takes place after the death or estrangement of $j, i \neq j$. By contrast, the second and the third are sources of interest. Moreover, the return increases with the love of the partner, while the interest is sensitive only to appeal (physical, intellectual, financial ...).

The following simplifying assumptions are also made. First, the appeals and the personalities of the two individuals do not vary in time: this rule out again, learning and adaptation processes which are often important over a long range of time [6, 7]. Thus, the model can only be used for short periods of time (months/years), for example in predicting if a love story will be characterized by regular or stormy feelings. Second, synergism is assumed to be negligible, i.e. Oblivion and return depend only upon one state variable. Finally, all mechanisms were supposed to be linear. The result was the following model:

$$
\begin{aligned}
& \frac{d x_{1}}{d t}=-\alpha_{1} x_{1}(t)+\beta_{1} x_{2}(t)+\gamma_{1} A_{2}, \\
& \frac{d x_{2}}{d t}=-\alpha_{2} x_{2}(t)+\beta_{2} x_{1}(t)+\gamma_{2} A_{1},
\end{aligned}
$$

where $\alpha_{i}, \beta_{i}$ and $\gamma_{i}$, as well as the appeals $A_{i}$, are constant and positive parameters. The negative term $-\alpha_{i} x_{i}(t)$, due to oblivion, says that the love of $i$, in the absence of the partner $j$, decays exponentially $x_{i}(t)=x_{i}(0) \exp \left(-\alpha_{i}(t)\right)$. The second term $\beta_{i} x_{j}(t)$ is the return, and the third $\gamma_{i} A_{j}$ is the reaction to the partner's appeal. Thus, each person is identified by four parameters: the appeal $A_{i}$, the forgetting coefficient $\alpha_{i}$ and the reactive-ness $\beta_{i}$ and $\gamma_{i}$ to the love and appeal of the partner.

Under suitable assumptions on the behavior of the individuals, the model turns out to be a positive linear enjoying, as such, a number of remarkable properties, which are in agreement with common wisdom on the argument. These properties are used to explore the consequences that individual behavior can have on the community structure. The main result along this line is that individual appeal is the driving force that creates order in the community.

Sprott J.C. [1] in his paper presented to the Chaos and Complex system seminar in Madison, Wisconsin on February 6, 2001 discussed various models of love and happiness. Some of the models described in the paper:

\section{Love model for Romeo and Juliet}

$$
\begin{aligned}
& \frac{d R}{d t}=a R+b J \\
& \frac{d J}{d t}=c R+d J
\end{aligned}
$$

where,

- $\mathrm{R}$ is Romeo's love for Juliet

- J is Juliet's love for Romeo (or hate if negative)

- a, b, c, d are constants that determine the "Romantic styles".

For different values of the constants there are several cases, but there are some limitations of the model:

1. It is difficult to quantify and measure love and hate.

2. Love is not a scalar (different types).

3. Parameters change in time and with the situation.

4. Parameters may be different for love and hate.

5. There are always other variables.

\section{Love Triangle Model}

Another model representing love triangle is also proposed by him

1. Romeo has a mistress, Guinevere.

2. Guinevere and Juliet don't know about one another. 
3. Romeo responds to each with the same romantic style (same a and b).

4. Guinevere's hate has the same effect on his feelings for Juliet as does Juliet's love, and vice versa.

\section{Love triangle equations}

$$
\begin{aligned}
& \frac{d R_{J}}{d t}=a R_{J}+b(J-G) \\
& \frac{d J}{d t}=c R_{J}+d J \\
& \frac{d R_{G}}{d t}=a R_{G}+b(G-J) \\
& \frac{d G}{d t}=e R_{G}+f G \\
& \bullet \quad \text { System is 4D (4 variables) } \\
& \bullet \quad \text { There are } 6 \text { parameters } \\
& \bullet \quad \text { System is is linear (no chaos) }
\end{aligned}
$$

J. Wauer et.al [8] studied human romantic relationships via system dynamics methodology. Starting point is a time-invariant linear model of two individuals without interaction with environment. Specifically, timedependent fluctuations both in the source terms and the system parameters are introduced and examined in their consequences where also more realistic non-linear modeling is proposed and analyzed. In reality, there are short- and long-termed fluctuations of personal feelings due to, for instance, biological cycles and varying stresses from the daily job. While it is straightforward to introduce such emotional patterns into the mathematical model, it is difficult to measure parameter values quantatively.

For couples with all kinds of exotic feeling, a neverending list of new phenomena may be expected. However, the variability is to be expected to be more limited for couples of cautious individuals. One interesting effect is the cyclic change of love about a stationary center of steady love for a couple governed by linear state equations, or a so-called robust couple governed by equations with nonlinear return functions. For a fragile couple, there is second stable fixed point of stationary hate, and the cyclic behavior may occur about either of the two centers. A more complex irregular motion pattern surrounding both centers with alternating love and hate of different time spans might appear during different time periods, but it seems very seldom for cautious lover with strong damping.

\section{Happiness Model}

A sequence of models for the time evolution of one's happiness in response to external events is described. These models with added nonlinearities can produce stable oscillations and chaos even without external events. Potential implications for psychotherapy and a personal approach to life are discussed. It was proved by Sprott [1] in this paper that even the simplest linear case, can exhibit temporal oscillations in their love for one another, so that Romeo's love $\mathrm{R}$ for Juliet is given by

$$
\frac{d^{2} x}{d t^{2}}+\beta \frac{d x}{d t}+\omega^{2} x=F(t)
$$

where,

$\beta$-Damping (rate of decay),

$\omega$-Natural resonant frequency of oscillation (in units of radians per unit time)

$F(t)$-External forces

$$
H=\frac{d x}{d t} \text { - Happiness }
$$

Without loss of generality, the frequency can be taken as $\omega=1$, which amounts to measuring time in units of $1 / \omega$. Equation (1) is the familiar expression for a damped (if $\beta>0$ ) harmonic oscillator describing many phenomena such as a mass on a spring, a smallamplitude pendulum, or a resonant electrical circuit. The feedback that allows oscillations is through Juliet's reaction to Romeo's love. This is the simplest dynamical model for Romeo's love and his resulting happiness.

\section{THE PROPOSED MODEL}

The model of Rinaldi [5] does not consider the effect of learning and adaptation and synergism after living together (in the case of arrange marriages in India, which have succeeded over centuries). This suggests that the emotional interaction of two individual must be considered in the modeling process.

Further, emotion of a particular individual with respect to other cannot increase infinitely. Hence emotions may vary; we assume that it is proportional to $X_{1} X_{2}$

Under the above assumption the following model is proposed

$$
\begin{aligned}
& \frac{d x_{1}}{d t}=-\alpha_{1} x_{1}+\beta_{1} x_{2}+A_{2}+d_{1} x_{1} x_{2} \\
& \frac{d x_{2}}{d t}=-\alpha_{2} x_{2}+\beta_{2} x_{1}+A_{1}+d_{2} x_{1} x_{2}
\end{aligned}
$$

where,

$x_{1}$ - measure of emotions of individual 1 .

$x_{2}$-measure of emotions of individual 2.

$\alpha, \beta, A$ and $d$ are constant and positive parameters. Now we will analyze (2) using equilibrium analysis

\section{SOLUTION OF THE PROPOSED MODEL}

The model is given as 


$$
\begin{aligned}
& \frac{d x_{1}}{d t}=-\alpha_{1} x_{1}+\beta_{1} x_{2}+A_{2}+d_{1} x_{1} x_{2} \\
& \frac{d x_{2}}{d t}=-\alpha_{2} x_{2}+\beta_{2} x_{1}+A_{1}+d_{2} x_{1} x_{2}
\end{aligned}
$$

The equilibrium points of the above system are solutions of

$$
\frac{d x_{1}}{d t}=0 \quad \text { and } \quad \frac{d x_{2}}{d t}=0
$$

Equation (4) gives

$$
\begin{aligned}
& x_{1}=\frac{1}{2\left(d_{2} \alpha_{1}+\beta_{2} d_{1}\right)}\left[-\left(A_{1} d_{1}+\beta_{1} \beta_{2}-A_{2} d_{2}-\alpha_{1} \alpha_{2}\right)\right. \\
& \pm\left\{\left(A_{2} d_{2}\right)^{2}+\left(\beta_{1} \beta_{2}\right)^{2}+\left(A_{1} d_{1}\right)^{2}+\left(\alpha_{1} \alpha_{2}\right)^{2}\right. \\
& -2 \beta_{1} \beta_{2} A_{1} d_{1}-2 A_{2} d_{2} \alpha_{1} \alpha_{2}-2 A_{1} d_{1} \alpha_{1} \alpha_{2} \\
& \left.\left.-2 \beta_{1} \beta_{2} A_{2} d_{2}-4 d_{1} \alpha_{2} A_{2} \beta_{2}-4 A_{1} \alpha_{1} \beta_{1} d_{2}\right\}^{1 / 2}\right]
\end{aligned}
$$

and

$$
\begin{aligned}
& x_{2}=\frac{1}{2\left(d_{1} \alpha_{2}+\beta_{1} d_{2}\right)}\left[-\left(A_{2} d_{2}+\beta_{1} \beta_{2}-A_{1} d_{1}-\alpha_{1} \alpha_{2}\right)\right. \\
& \pm\left\{\left(A_{2} d_{2}\right)^{2}+\left(\beta_{1} \beta_{2}\right)^{2}+\left(A_{1} d_{1}\right)^{2}+\left(\alpha_{1} \alpha_{2}\right)^{2}\right. \\
& -2 \beta_{1} \beta_{2} A_{1} d_{1}-2 A_{2} d_{2} \alpha_{1} \alpha_{2}-2 A_{1} d_{1} \alpha_{1} \alpha_{2} \\
& \left.\left.-2 \beta_{1} \beta_{2} A_{2} d_{2}-4 d_{1} \alpha_{2} A_{2} \beta_{2}-4 A_{1} \alpha_{1} \beta_{1} d_{2}\right\}^{1 / 2}\right]
\end{aligned}
$$

Substituting the given values in the above ${ }^{X_{1}}$ and $X_{2}$

$$
\begin{aligned}
& k=A_{1} d_{1}+\beta_{1} \beta_{2}-A_{2} d_{2}-\alpha_{1} \alpha_{2} \\
& l=\left[\left(A_{2} d_{2}\right)^{2}+\left(\beta_{1} \beta_{2}\right)^{2}+\left(A_{1} d_{1}\right)^{2}+\left(\alpha_{1} \alpha_{2}\right)^{2}\right. \\
& -2 \beta_{1} \beta_{2} A_{1} d_{1}-2 A_{2} d_{2} \alpha_{1} \alpha_{2}-2 A_{1} d_{1} \alpha_{1} \alpha_{2} \\
& \left.-2 \beta_{1} \beta_{2} A_{2} d_{2}-4 d_{1} \alpha_{2} A_{2} \beta_{2}-4 A_{1} \alpha_{1} \beta_{1} d_{2}\right]^{1 / 2} \\
& m=2\left(d_{1} \alpha_{2}+\beta_{1} d_{2}\right) \\
& n=2\left(d_{2} \alpha_{1}+\beta_{2} d_{1}\right) \\
& k, l, m, n>0
\end{aligned}
$$

We get,

$$
x_{1}=\frac{-k \pm l}{m}
$$

and

$$
x_{2}=\frac{k \pm l}{n}
$$

\section{STABILITY ANALYSIS}

In order to study stability of these equilibrium points we use some assumptions:

(1) $\alpha_{1} \alpha_{2}>2 \beta_{1} \beta_{2}$

(2) $A_{1} d_{1}>2 A_{2} d_{2}$
On observing the values of $x_{1}$ and $x_{2}$ we get that there are four cases:

1. Case 1:

Let us consider the values

$$
\begin{aligned}
& x_{1}=\frac{-k+l}{m}, \\
& x_{2}=\frac{k+l}{n}
\end{aligned}
$$

For stability analysis we have,

$$
X_{1}=x_{1}-\left(\frac{-k+l}{m}\right)
$$

and

$$
X_{2}=x_{2}-\left(\frac{k+l}{n}\right)
$$

Substituting these values in the original model and linearizing we get,

$$
\frac{d^{2} X_{1}}{d t^{2}}+a_{1} \frac{d X_{1}}{d t}+a_{2} X_{1}+r=0
$$

where,

$$
\begin{aligned}
& a_{1}=\alpha_{1}-d_{1}\left(\frac{k+l}{n}\right) \\
& a_{2}=-\beta_{1}^{2}-\beta_{1} d_{2}\left(\frac{l-k}{m}\right) p+\beta_{1} \alpha_{2} p \\
& +d_{1} \alpha_{2} p\left(\frac{l-k}{m}\right)-\beta_{1} d_{2} p\left(\frac{k+l}{n}\right)-\beta_{1} d_{1} p\left(\frac{l-k}{m}\right) \\
& -d_{1} d_{2}\left(\frac{k+l}{n}\right)\left(\frac{l-k}{m}\right)-d_{1} d_{2} p\left(\frac{l-k}{m}\right)^{2}
\end{aligned}
$$

It can be calculated that,

$$
a_{1}<0 \quad \text { and } \quad a_{2}<0
$$

Using Routh-Hurwitz criteria we get,

$$
\left[\begin{array}{ll}
a_{1} & 1 \\
0 & a_{2}
\end{array}\right]
$$

Therefore by (8) and (9),

$$
a_{1} a_{2}>0
$$

Hence the equilibrium points given by equation (7) are unstable.

Further it may be calculated that if

(1) $d_{1}>d_{2}$ and

(2) $p=\frac{x_{2}}{x_{1}}>1$ 
Then,

$a_{2}>0$

Therefore,

$a_{1} a_{2}<0$

Hence we get asymptotically stable system.

\section{Case 2}

Let us consider the values

$x_{1}=\frac{-k-l}{m}$,

$x_{2}=\frac{k+l}{n}$

For stability analysis we have,

$$
X_{1}=x_{1}-\left(\frac{-k-l}{m}\right) \text {, }
$$

and

$$
X_{2}=x_{2}-\left(\frac{k+l}{n}\right)
$$

Substituting these in the original model and linearizing we get,

$$
\frac{d^{2} X_{1}}{d t^{2}}+a_{1} \frac{d X_{1}}{d t}+a_{2} X_{1}+r=0
$$

where,

$$
\begin{aligned}
& a_{1}=\alpha_{1}-d_{1}\left(\frac{k+l}{n}\right) \\
& a_{2}=-\beta_{1}^{2}-\beta_{1} d_{1}\left(\frac{k+l}{n}\right)^{2}+\beta_{1} d_{1}\left(\frac{k+l}{m}\right) \\
& +d_{1}^{2}\left(\frac{k+l}{n}\right)^{2}+\beta_{1} \alpha_{2} p+\beta_{1} d_{2} p\left(\frac{k+l}{m}\right) \\
& -d_{1} \alpha_{2} p\left(\frac{k+l}{m}\right)-d_{1} d_{2} p\left(\frac{k+l}{m}\right)^{2}
\end{aligned}
$$

It can be calculated that,

$$
a_{1}<0 \quad \text { and } \quad a_{2}<0
$$

Using Routh-Hurwitz criteria we get

$$
\left[\begin{array}{cc}
a_{1} & 1 \\
0 & a_{2}
\end{array}\right]
$$

Therefore by (11) and (12),

$$
a_{1} a_{2}>0
$$

Hence the equilibrium points given by equation (10) are unstable.

Further it may be calculated that if

$$
d_{1}>p\left(\alpha_{2}+d_{2}\right)
$$

Then,

$$
a_{2}>0
$$

Therefore,

$a_{1} a_{2}<0$

Hence we get asymptotically stable system.

3. Case 3:

Let us consider the values

$$
\begin{aligned}
& x_{1}=\frac{-k+l}{m}, \\
& x_{2}=\frac{k-l}{n}
\end{aligned}
$$

For stability analysis we have,

$$
X_{1}=x_{1}-\left(\frac{-k-l}{m}\right) \text {, }
$$

and

$$
X_{2}=x_{2}-\left(\frac{k+l}{n}\right)
$$

Substituting these values in the original model and linearizing we get,

$$
\frac{d^{2} X_{1}}{d t^{2}}+a_{1} \frac{d X_{1}}{d t}+a_{2} X_{1}+r=0
$$

where,

$$
\begin{aligned}
& a_{1}=\alpha_{1}-d_{1}\left(\frac{k-l}{n}\right) \\
& a_{2}=-\beta_{1} \alpha_{2} p-\beta_{1} d_{2} p\left(\frac{l-k}{n}\right)+d_{1} \alpha_{2} p\left(\frac{l-k}{m}\right) \\
& -d_{1} d_{2} p\left(\frac{l-k}{m}\right)^{2}-\beta_{1}^{2}+\beta_{1} d_{2}\left(\frac{l-k}{n}\right) \\
& -d_{1} \beta_{1} p\left(\frac{l-k}{m}\right)+d_{1} d_{2} p\left(\frac{l-k}{m n}\right)^{2}
\end{aligned}
$$

It can be calculated that,

$$
a_{1}>0 \quad \text { and } \quad a_{2}<0
$$

Using Routh-Hurwitz criteria we get,

$$
\left[\begin{array}{cc}
a_{1} & 1 \\
0 & a_{2}
\end{array}\right]
$$

Therefore by (14) and (15),

$$
a_{1} a_{2}<0
$$

Hence, the system is asymptotically stable.

4. Case4:

Let us consider the values 


$$
\begin{aligned}
& x_{1}=\frac{-k-l}{m}, \\
& x_{2}=\frac{k-l}{n}
\end{aligned}
$$

For stability analysis we have,

$$
X_{1}=x_{1}-\left(\frac{-k-l}{m}\right) \text {, }
$$

and

$$
X_{2}=x_{2}-\left(\frac{k+l}{n}\right)
$$

Substituting these values in the original model and linearizing we get,

$$
\frac{d^{2} X_{1}}{d t^{2}}+a_{1} \frac{d X_{1}}{d t}+a_{2} X_{1}+r=0
$$

where,

$$
\begin{aligned}
& a_{1}=\alpha_{1}-d_{1}\left(\frac{k-l}{n}\right) \\
& a_{2}=\beta_{1} \alpha_{2} p+\beta_{1} d_{2} p\left(\frac{k+l}{m}\right)-d_{1} \alpha_{2} p\left(\frac{k+l}{m}\right) \\
& -d_{1} d_{2} p\left(\frac{k+l}{m}\right)^{2}-\beta_{1}^{2}-d_{1}^{2}\left(\frac{l-k}{n}\right)\left(\frac{l+k}{m}\right)
\end{aligned}
$$

It can be calculated that,

$$
a_{1}>0 \quad \text { and } \quad a_{2}<0
$$

Using Routh-Hurwitz criteria we get

$$
\left[\begin{array}{cc}
a_{1} & 1 \\
0 & a_{2}
\end{array}\right]
$$

Therefore by (17) and (18),

$$
a_{1} a_{2}<0
$$

Hence, the system is asymptotically stable.

It is clear that all the four cases are giving as ymptotically stable system. Hence the proposed model results in asymptotically stable system.

\section{CONCLUSIONS}

The determination of the behavioral parameters is undoubtedly a difficult task, although some studies on attachment styles [9, 10, 11] suggest ways of identifying categories of individuals with high or low reactive-ness or forgetting coefficients.

The model considered in this study has remarkable properties since it is asymptotically stable. In the present study, the necessary and sufficient conditions for asymptotic stability are

(1) $\beta_{1} \beta_{2}<\alpha_{1} \alpha_{2}$,
(2) $A_{1} d_{1}>A_{2} d \quad\left(=\frac{A_{1}}{A_{2}}>\frac{d_{1}}{d_{2}}\right)$

(1) tells that the system is asymptotically stable if the (geometric) mean reactive-ness to love $\sqrt{\beta_{1} \beta_{2}}$ is smaller than the (geometric) mean forgetting coefficient $\sqrt{\alpha_{1} \alpha_{2}}$.

(2) tells that the system is asymptotically stable if the ratio of appeals $\frac{A_{1}}{A_{2}}$ is greater than the reciprocal of ratio of mutual intensiveness coefficient $\frac{d_{1}}{d_{2}}$.

The stability of proposed model shows that the effect of learning and adaptation and synergism after living together is an important factor. Hence it can be concluded that in arrange marriages the emotional interaction of two individuals plays an important role in modeling. Although it is clear that emotion of a particular individual with respect to other cannot increase infinitely. But there is existence of learning and adaptation.

\section{REFERENCES}

[1] J.C.Sprott "Dynamics of love and Happiness", Chaos and Complex Systems Seminar in Madison, Wisconsin 2001.

[2] Stenberg, R.J. \& Barnas, M.L. (Eds.). The psychology of love. New Haven, CT: Yale University Press 1988.

[3] S.H.Srogatz, Nonlinear Dynamics and Chaos with Applications to Physics, Biology, Chemistry and Engineering, Addison-Wesley, Reading, M.A. 1994.

[4] S.H.Strogatz. Love affairs and differential equations, math.Mag. 1988; 61: 35.

[5] Sergio Rinaldi , "Love dynamics", 1998; 95: 181192.

[6] R. R. Kobak, C. Hazan. Attachment in marriage: the effect of security and accuracy of working models, J. Personality Social Psych. 1991; 60: 861-869.

[7] E. Scharfe, K. Bartholomew, Realiability and stability of adult attachment patterns, Personal Relationship 1994; 1: 23-43.

[8] J. Wauer, D. Schwarzer, G.Q. Cai, Y.K. Lin, "Dynamical models of love with time-varying fluctuations" 2007; 188: 1535-1448.

[9] K. Bartholomew, L. M. Horowitz. Attachment styles among young adults: a test of a four-category model, J. Personality Social Psych. 1991; 61: 226-244

[10] K.B. Carnelly, R. Janoff-Bulman. Optimism about love relationships: general vs. specific lessons from one's personal experiences, J. Social Personal Relat. 1992; 9: 5-20.

[11]D.W. Griffin, K. Bartholomew, Models of the self and other. Fundamental dimensions underlying measures of adult attachment, J. Personality Social Psych. 1994; 67: 430-445. 
Dharna Satsangi has earned integrated M.Sc.-M. Phil. in Mathematics with specialization in Computer Science from the Dayalbagh Educational Institute, India (2009) and is currently pursuing Ph.D. at the same department. Her research includes mathematical models of biological systems, psychological behavior of humans and graph layout problems.

Prof. Arun K. Sinha has earned M. Sc. in Mathematics Specialization in Statistics and Ph.D. in Mathematics from Agra College, India. His research areas are statistics and bio-mathematics. He has 35 years of teaching experience. Currently he is Head, Department of Mathematics, Dayalbagh Educational Institute, Agra. 